EDITORIAL

\title{
Editor's Introduction to This Issue
}

\author{
Yeun-Jun Chung* \\ IRCGP, The Catholic University of Korea, College of Medicine, Seoul 06591, Korea
}

In this issue, diverse topics of genome biology and informatics, such as non-coding RNAs, biological implication of introns, transcriptome sequencing, diseaseassociation of genetic variants, oncogenomics, genetic evolution, and analysis of promoter regions are published. Drs. Yong Sun Lee and Xiaoyong Bao's group (University of Texas) reviewed the biogenesis and functions of tRNAderived RNA Fragments (tRFs). tRFs is a newly introduced class of non-coding RNAs. Considering that tRFs are not random degradation products but are functional non-coding RNAs through specific tRNA cleavage, their review must be helpful to understand this emerging class of non-coding RNA. Dr. Sun Shim Choi's group (Kangwon National University) raised interesting questions about intron. For examples, why only eukaryotes have introns or why the amount of total introns varies in different species and in different genes? In their review, they summarized previous researches about the functional roles or benefits of introns and suggest their view. Transcriptome sequencing is now one of the common and clinically applicable genome analyses tools. In this issue, Dr. Sangwoo Kim's group (Yonsei University) introduced the routine workflow of transcriptome sequencing workflow together with related software, focusing particularly on transcriptome reconstruction and expression quantification. There are three papers regarding genetic evolution in diverse species. Dr. Kornsorn Srikulnath's group (Kasetsart University) reviewed the role of chromosome changes in crocodilian evolution and diversity. Dr. Heebal Kim's group (Seoul National University) reported the genes related to positive selection in commercial pig breeds and also estimated the heritability in Holstein breeding. For further details, please visit G\&I homepage (http://www.kogo.or.kr/webapp/kogo_publish/ genomics_and_informatics/).

*Corresponding author: Tel: +82-2-2258-7343, Fax: +82-2-537-0572, E-mail: yejun@catholic.ac.kr

Copyright $(2015$ by the Korea Genome Organization

(c) It is identical to the Creative Commons Attribution Non-Commercial License (http://creativecommons.org/licenses/by-nc/4.0/). 